\title{
Impact Vibration Analysis of Group of Hexagonal Bars Immersed in Liquid*
}

\author{
Toshihiko HORIUCHI**
}

\begin{abstract}
A simulation method was developed to calculate the vibrational response of a group of hexagonal bars installed in a restraint immersed in liquid during seismic excitation. The influence of fluid force on structural motion was modeled using an added-mass matrix. This matrix was then transformed into the space composed of the eigenmodes of the hexagonal bars without added mass and used for eigenvalue analysis of the entire bar-group structure. This method reduced the computational time needed for practical added-mass matrix calculation and the eigenvalue analysis. It was shown that the proposed method yielded almost the same eigenvalues as the conventional method conducted in standard physical space. Added-mass models for use in impactvibration analysis were investigated using the proposed method. Comparing the results calculated using the proposed method with those calculated using concentrated added mass, which is a simplified model, showed that the latter can be used for a rough response calculation, while the precise calculation requires the added-mass matrix method.
\end{abstract}

Key Words: Nonlinear Vibration, Vibration Coupled with Fluid Motion, Seismic Motion, Eigenvalue Analysis, Simulation, Bar Group, Hexagonal Bar, Impact

\section{Introduction}

Development of a method of analysis for seismic response of a structural system is important for the design of the system, in order to ensure its integrity. For this reason, various research has been conducted to develop such methods for a group of bars such as those that form the core components of nuclear plants $^{(1)-(6)}$. A group of bars is composed of a large number of bars which are installed close together. Due to number of bars, a large number of degrees of freedom (DOFs) are required in calculation in order to simulate the motions of the structure. In addition, impact may take place between adjacent fuel assemblies when an earthquake excites such structures as the core components of fast breeder reactors (FBR) and pressurized water reactors (PWR). Hence, impact forces should be considered in seismic analyses

* Received 7th April, 1995. Japanese original: Trans. Jpn. Soc. Mech. Eng., Vol. 60, No. 576, C(1994), pp. 2691-2698. (Recieved 22nd September, 1993)

** Mechanical Engineering Research Laboratory, Hitachi, Ltd., 502 Kandatsu, Tsuchiura, Ibaraki 300 , Japan of such structural systems. Since each impact usually occurs over a very short duration, a small time step should be used in time-history analysis. Therefore, calculation techniques providing a reasonable reduction in the number of DOFs and fast time-integral computation are required. One method for this purpose is the pseudo-external force method ${ }^{(7)}$, in which nonlinear forces are treated as external forces. In addition, when a structure is immersed in liquid, it is necessary to take the force caused by reaction of the fluid (fluid force) on the structure into consideration. In response analysis, fluid force is usually taken into account using added mass because it is generally proportional to structural acceleration. Various studies have been made to evaluate the added mass of a group of bars ${ }^{(8)-(12)}$. Since bars in a liquid vibrate under fluid-force coupling, evaluation of added mass is also important in impact-vibration analysis. This calculation also requires a large number of DOFs. Therefore, it is desirable to reduce this number of DOFs.

The author has proposed a method of impactvibration analysis for groups of bars in a single row ${ }^{(13)}$, and for groups of hexagonal bars in a hexagonal 
$\operatorname{grid}^{(14)}$, in which impact force is considered as pseudoexternal force and can be determined without iteration. This report describes a way of introducing added mass into the proposed impact-vibration analy. sis method in order to expand the applicability of the method to groups of bars immersed in liquid, such as in the case of core components of nuclear power plants. Also, the influence of added-mass modeling on response calculation results will be investigated using the proposed method.

\section{Analysis Method}

\subsection{Equation of motion and method of analysis for impact vibration}

Here, we consider a group of hexagonal bars installed in a tank, as shown in Fig. 1. Each bar is numerically modeled using finite beam elements in which each node has deflectional and rotational freedom with respect to both the $X$ - and $Y$-axes as shown in Fig. 2. Impact is assumed to take place only

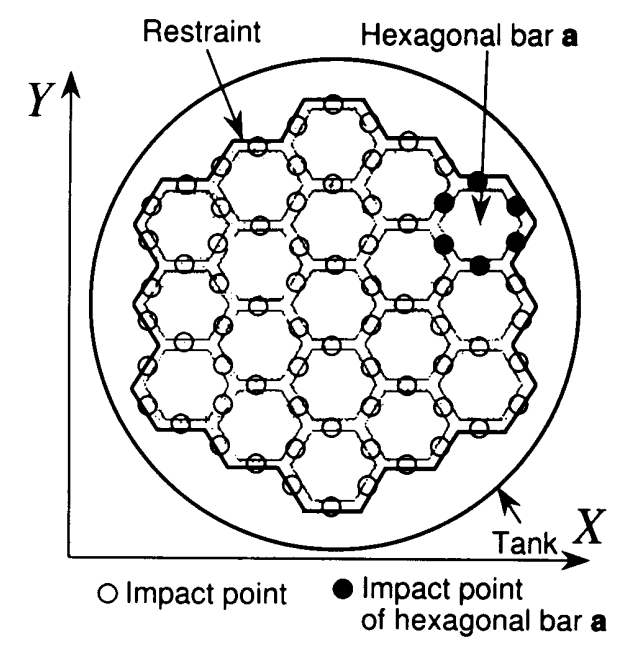

Fig. 1 The group of hexagonal bars under consideration

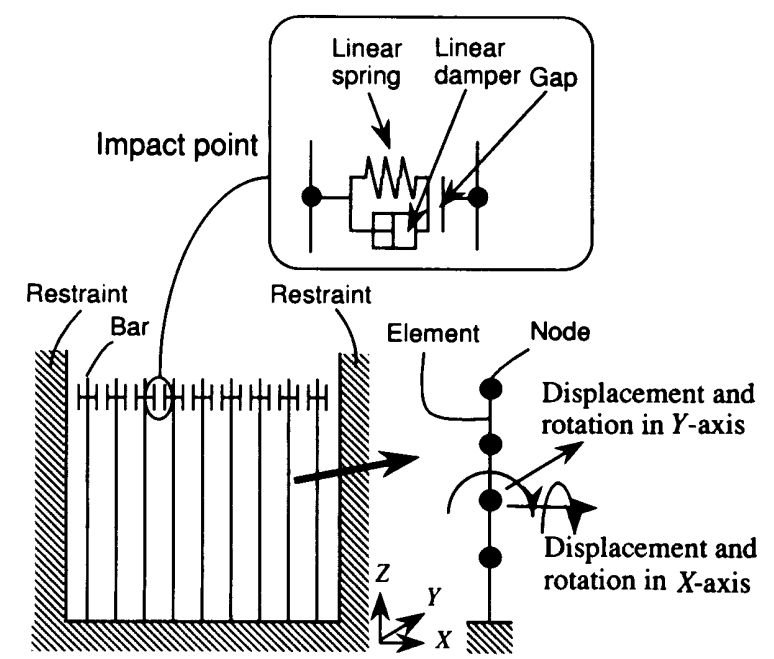

Fig. 2 Numerical model of a group of hexagonal bars at impact points, which are pairs of nodes in two adjacent bars. Since the bar under consideration has a hexagonal cross section, one node can have six impact points as shown in Fig. 1. A bar may have more than one impact-point node.

Fluid force vector $\hat{\boldsymbol{q}}_{s}$ is assumed to be the product of the absolute acceleration vector of the structure $\ddot{\vec{x}}$, which includes the DOFs of the tank, and the added mass matrix, $\widehat{\boldsymbol{M}}_{v}$, so that

$$
\widehat{\boldsymbol{q}}_{f}=-\widehat{\boldsymbol{M}}_{v} \ddot{\overline{\boldsymbol{x}}}
$$

The matrix $\hat{\boldsymbol{M}}_{v}$ can be calculated, for example, using the finite element method based on velocity-potential theor $\mathrm{y}^{(15)}$. It should be noted that the above equation is not, strictly speaking, exact for the present analysis because deflections are assumed to be small in the theory. However, Eq. (1) can be used as an approximation since the displacement of the bars is limited by the restraints, and since the results of analysis using this method show good agreement with experimental results, as the author has reported ${ }^{(16)}$. It should also be noted that it is necessary to calculate the matrix $\overline{\boldsymbol{M}}_{v}$ using a three-dimensional model when the axis length is relatively small compared to the radius of the group of bars; in this case the distribution of added mass cannot be neglected ${ }^{(16),(17)}$.

When the tank is rigid and therefore its motion is the same as that of the base, the absolute- acceleration vector, $\ddot{\bar{x}}$, can be written as

$$
\ddot{\ddot{x}}^{T}=\left\{\ddot{\boldsymbol{x}}^{T}, \boldsymbol{o}^{T}\right\}-\left\{\left(\boldsymbol{I}_{X Y} \ddot{\boldsymbol{x}}_{0}\right)^{T}, \ddot{\boldsymbol{x}}_{0}{ }^{T}\right\}
$$

where

$\ddot{x}^{T}$ : the vector of acceleration of the DOFs other than those of the tank relative to the base,

$\ddot{x}_{0}^{T}=\left\{\ddot{x}_{0}, \ddot{y}_{0}\right\}$,

$\ddot{x}_{0}$ : the acceleration of the base in the $X$ direction,

$\ddot{y}_{0}$ : the acceleration of the base in the $Y$ direction,

$\boldsymbol{I}_{X Y}=\left[\boldsymbol{i}_{X}, \boldsymbol{i}_{Y}\right]$

$\boldsymbol{i}_{u}$ : the vector for which all components with respect to the $u$ direction are one and others are zero, $(u$ is $X$ or $Y$ ),

$\boldsymbol{o}^{T}=\{0,0\}$.

When the vector $\ddot{x}$ is $n$-dimensional, describing the tank-motion terms separately from the others, the matrix $\hat{\boldsymbol{M}}_{v}$ becomes

$$
\widehat{\boldsymbol{M}}_{v}=\left[\begin{array}{ll}
\boldsymbol{M}_{v} & \boldsymbol{M}_{v 0} \\
\boldsymbol{M}_{v 0}^{T} & \boldsymbol{M}_{v 00}
\end{array}\right]
$$

Substituting Eqs. (2) and (3) into Eq. (1) gives the fluid-force vector $\boldsymbol{q}_{f}$ with respect to the non-tank components, as follows :

$$
\begin{aligned}
\boldsymbol{q}_{f} & =-\boldsymbol{M}_{v} \ddot{\boldsymbol{x}}+\left(\boldsymbol{M}_{v} \boldsymbol{I}_{X Y}+\boldsymbol{M}_{v 0}\right) \ddot{\boldsymbol{x}}_{0} \\
& =-\boldsymbol{M}_{v} \ddot{\boldsymbol{x}}+\boldsymbol{M}_{0} \boldsymbol{I}_{X Y} \ddot{\boldsymbol{x}}_{0}
\end{aligned}
$$

The first term on the right-hand side of Eq. (4) is the 
force coming from the added mass. The matrix $\boldsymbol{M}_{v}$ will be referred to as the physical-space added mass. The second term is similar to a buoyancy force in the vertical direction. By introducing the nonlinear impact force as a pseudo-external force and by expressing the fluid force using Eq. (4), the following formula for the equation of motion in terms of the vector $x$ of displacement relative to the base is obtained.

$$
\left(\boldsymbol{M}+\boldsymbol{M}_{v}\right) \ddot{\boldsymbol{x}}+\boldsymbol{C} \dot{\boldsymbol{x}}+\boldsymbol{K} \boldsymbol{x}=\boldsymbol{f},
$$

where

$\boldsymbol{M}:$ the mass matrix,

$C$ : the damping matrix,

$K$ : the stiffness matrix,

$x$ : the vector of displacement relative to the base,

$\boldsymbol{f}:$ an external force vector.

The external force vector is a summation of the inertia-force vector caused by the base acceleration and the impact-force vector, $\boldsymbol{q}_{c}$; therefore, it can be written as

$$
\boldsymbol{f}=-\left(\boldsymbol{M}-\boldsymbol{M}_{0}\right) \boldsymbol{I}_{X Y} \ddot{\boldsymbol{x}}_{0}+\boldsymbol{q}_{c} .
$$

Based on the equation of motion, Eq. $(5)$, the eigenmodes of an entire group of bars can be calculated, and the analysis of the impact vibration of an entire group of bars becomes possible by means of a method that the author has previously reported ${ }^{(14)}$, as follows. First, the eigenmode matrix, $\Phi=\left[\phi_{1}, \phi_{2}, \cdots\right.$, $\boldsymbol{\phi}_{m}$ ], is obtained by assuming that $\boldsymbol{C}=0$ and $\boldsymbol{f}=0$, where $m$ is the number of degrees of freedom. Then, assuming that the damping matrix can be diagonal. ized by the eigenmode matrix, the equation of motion is transformed into

$$
\ddot{u}_{n}+2 \zeta_{n} \omega_{n} \dot{u}_{n}+\omega_{n}^{2} u_{n}=\bar{a}_{n},
$$

where

$u_{n}:$ the $n$th modal displacement,

$\omega_{n}:$ the $n$th modal eigen circular frequency,

$\zeta_{n}:$ the $n$th modal damping ratio,

$\bar{a}_{n}:$ the $n$th modal external force,

$$
=\boldsymbol{\phi}_{n}^{T} \boldsymbol{f} / \boldsymbol{\phi}_{n}^{T}\left(\boldsymbol{M}+\boldsymbol{M}_{v}\right) \boldsymbol{\phi}_{n} \text {. }
$$

Finally, the vibration response of the group of bars can be calculated by transforming the responses of the single-degree-of-freedom systems in the modal space back into physical space, that is,

$$
\boldsymbol{x}=\sum_{n=1}^{m} \boldsymbol{\phi}_{n} u_{n}=\boldsymbol{\Phi} \boldsymbol{u},
$$

where $\boldsymbol{u}^{T}=\left\{u_{1}, u_{2}, \cdots, u_{m}\right\}$.

The number of DOFs may be reduced by eliminating high-order modes which make little contribution to the vibration response in Eq. ( 8 ).

\section{2 Method of eigenvalue analysis}

The vibration- ${ }^{-}$esponse analysis requires that the eigenfrequencies, the eigenmodes and so on be obtained through eigenvalue analysis. However, a group of bars has a large number of DOFs as mentioned earlier, and therefore much computational effort is required-not only for response analysis but also for physical-space added-mass matrix calculation and eigenvalue analysis. To solve this problem, a reduction in the number of DOFs in the entire displacement vector may be accomplished-not by eliminating high-order modes after eigenvalue analysis, but by reducing the number of DOFs in the displacement vector in the physical space before the eigenvalue analysis, in the following way. In the equation of motion, the displacement vector $\overline{\boldsymbol{x}}_{i}$ of the $i$ th bar is assumed to be arrayed sequentially in the vector $\boldsymbol{x}$, that is,

$$
\boldsymbol{x}^{T}=\left\{\overline{\boldsymbol{x}}_{1}^{T}, \cdots, \overline{\boldsymbol{x}}_{i}^{T}, \cdots, \overline{\boldsymbol{x}}_{N}^{T}\right\},
$$

where $N$ is the number of bars.

The vector $\overline{\boldsymbol{x}}_{i}$ can be written using the mode matrix of the $i$ th bar, $\overline{\boldsymbol{\Phi}}_{i}$, and the mode displacement vector, $\overline{\boldsymbol{w}}_{i}$, as follows,

$$
\overline{\boldsymbol{x}}_{i}=\overline{\boldsymbol{\Phi}}_{i} \overline{\boldsymbol{w}}_{i}=\left[\overline{\boldsymbol{\phi}}_{i_{1}}, \cdots, \overline{\boldsymbol{\phi}}_{i M}\right] \overline{\boldsymbol{w}}_{i},
$$

where $M$ is the number of DOFs of the bar. Then, when the required number of DOFs is $M^{\prime},\left(M^{\prime} \leq M\right)$, Eq. (10) can be expressed approximately using the reduced-mode matrix ( $M^{\prime}$ modes) $\overline{\boldsymbol{\Phi}}_{i}^{\prime}$ and the reduced displacement vector, $\overline{\boldsymbol{w}}_{i}^{\prime}$, as

$$
\overline{\boldsymbol{x}}_{i} \approx \overline{\boldsymbol{\Phi}}_{i}^{\prime} \overline{\boldsymbol{w}}_{i}^{\prime}=\left[\overline{\boldsymbol{\phi}}_{i 1}, \cdots, \overline{\boldsymbol{\phi}}_{i^{\prime}}\right] \overline{\boldsymbol{w}}_{i}^{\prime} \text {. }
$$

Applying this procedure to the displacement vector of the entire group of bars $\boldsymbol{x}$ gives

$$
\boldsymbol{x}=\left[\begin{array}{ccc}
\overline{\boldsymbol{\Phi}}_{1}^{\prime} & & \boldsymbol{O} \\
& \ddots & \\
\boldsymbol{O} & & \overline{\boldsymbol{\Phi}}_{N}^{\prime}
\end{array}\right]\left\{\begin{array}{c}
\overline{\boldsymbol{w}}_{1}^{\prime} \\
\vdots \\
\overline{\boldsymbol{w}}_{N}^{\prime}
\end{array}\right\}=\overline{\boldsymbol{\Phi}} \boldsymbol{w} .
$$

Substituting the above expression into the equation of motion yields the reduced equation of motion in terms of the modal displacement vector $\boldsymbol{w}$ :

$$
\boldsymbol{M}_{s} \ddot{\boldsymbol{w}}+\boldsymbol{C}_{s} \dot{\boldsymbol{w}}+\boldsymbol{K}_{s} \boldsymbol{w}=\boldsymbol{f}_{s},
$$

where

$$
\begin{aligned}
\boldsymbol{M}_{S} & =\overline{\boldsymbol{\Phi}}^{T} \boldsymbol{M} \overline{\boldsymbol{\Phi}}+\overline{\boldsymbol{\Phi}}^{T} \boldsymbol{M}_{v} \overline{\boldsymbol{\Phi}}, \\
\boldsymbol{C}_{S} & =\overline{\boldsymbol{\Phi}}^{T} \boldsymbol{C} \overline{\boldsymbol{\Phi}}, \\
\boldsymbol{K}_{S} & =\overline{\boldsymbol{\Phi}}^{T} \boldsymbol{K} \overline{\boldsymbol{\Phi}}
\end{aligned}
$$

and

$$
\boldsymbol{f}_{s}=\overline{\boldsymbol{\Phi}}^{T} \boldsymbol{f}
$$

It should be noted that $\boldsymbol{K}_{s}$ becomes a diagonal matrix ; however, for $\boldsymbol{M}_{s}$ that is not the case. Therefore Eq. (13) becomes a set of simultaneous equations. By the eigenvalue analysis associated with Eq. (13), the eigenparameters of the entire structure such as the eigenfrequencies $\omega_{i},\left(i=1, \cdots,\left(M^{\prime} \times N\right)\right)$ and the eigenmode matrix, $\boldsymbol{\Psi}$, can be calculated. Consequently, Eq. (5) can be transformed into the single-degree- of freedom expression in Eq. ( 7 ) via Eq. (13). Note that the eigenmode matrix in $\mathrm{Eq}_{\mathrm{q}}(\mathrm{8}), \boldsymbol{\Phi}$, is

$$
\boldsymbol{\Phi}=\overline{\boldsymbol{\Phi}} \boldsymbol{\Psi}
$$

Also note that the damping matrix $C$ is not necessarily 
diagonalized by the mode matrix $\boldsymbol{\Phi}$. However, the modes may be assumed to be independent, because the coupling effect of the damping matrix is usually small.

As observed in Eq. (14), the analysis based on Eq. (13) requires the modal-space added mass, $\overline{\boldsymbol{\Phi}}^{T} \boldsymbol{M}_{v} \overline{\boldsymbol{\Phi}}$, which is the added mass matrix with respect to a vector composed of the eigenmodes of the bar. The calculation of this matrix may be conducted using not the added-mass matrix with respect to all nodes used in vibration analysis as in Eq. (5), but, rather, using an added-mass matrix based on a fluid-region finite element model which can express at least those eigenmodes used in vibration analysis. Using the method discussed above, the computational load not only of the eigenvalue analysis but also of the added-mass analysis, may be reduced.

\subsection{Calculation examples using the proposed method}

2.3.1 Analysis of a single bar By conducting eigenvalue analysis of one hexagonal bar in a liquid, as shown in Fig. 3, we investigated how modeling of the fluid region in added mass calculation influences eigenvalue analysis results.

The bar has a length of $1000 \mathrm{~mm}$ and a minimum diameter (between two opposite sides) of $60 \mathrm{~mm}$, and is installed in a tank and immersed in a liquid. The distance between a side of the bar and the tank wall is $10 \mathrm{~mm}$. The structural boundary conditions are free

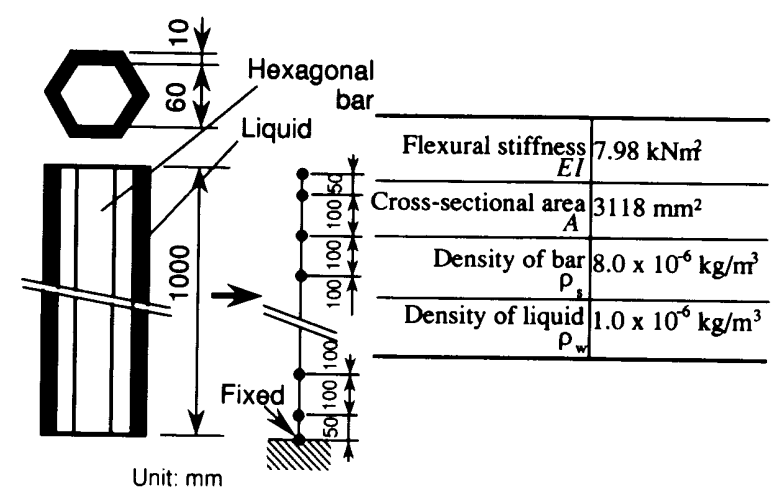

Fig. 3 Model of the bar

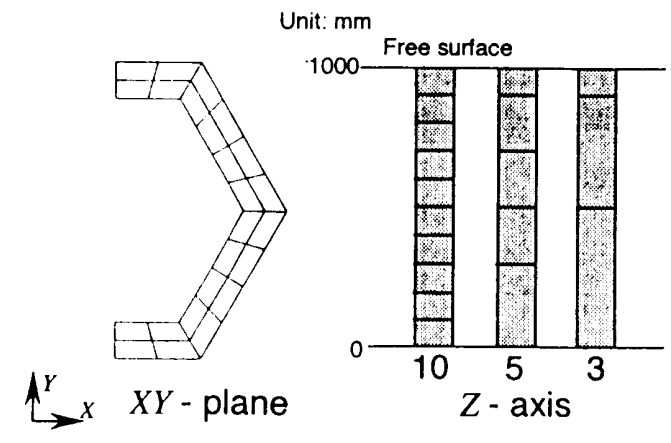

Fig. 4 Model of fluid region at the upper end and fixed at the lower end. Other parameters concerning the calculation are given in Fig. 3. With these parameters, the bar has a firstmode natural frequency of $10 \mathrm{~Hz}$ when it is in air. In the calculation of the added-mass matrix, three resolutions of finite-element modeling are considered, each having a different number of divisions along the $Z$-axis: 10,5 , or 3 . The positions of the divisions for the 5-division and 3-division models are determined so that they are close to the nodes of the first-through third or first-through-fifth eigenmode shapes, respectively. Other modeling conditions of the fluid region are as shown in Fig. 4 ; that is, the boundary conditions are set as: a free-surface condition at the level of the top of the bar, and zero velocity at the wall.

Table 1 shows results of the eigenvalue analysis, such as the eigenfrequencies and the participation factors to the excitation of the base, which is defined so that the mode amplitude at the upper end is one. Using the 10-division model, the physical-space addedmass analysis and the modal-space added-mass analysis produced almost the same results. In the case of the 5-division model, close results were obtained, although errors were relatively large in the higher modes. On the other hand, with the 3-division model, only the results of up to the third mode were good; the others showed large errors. These results show that the modal-space added-mass analysis gives eigenvalues as accurate as those of the physical-space added-mass analysis, when the necessary eigenmode shapes of the bar are considered in dividing the fluid region into finite elements.

Table 1 Eigenfrequency and participation factor of one bar

\begin{tabular}{|c|c|c|c|c|c|}
\hline Mode No. & $1 \mathrm{st}$ & 2nd & $3 \mathbf{r d}$ & 4 th & 5 th \\
\hline In air & $\begin{array}{l}9.99 \\
(1.57)\end{array}$ & $\begin{array}{c}62.7 \\
(-0.87)\end{array}$ & $\begin{array}{l}175.5 \\
(0.51)\end{array}$ & $\begin{array}{c}344.1 \\
(-0.36)\end{array}$ & $\begin{array}{l}569.7 \\
(-0.28)\end{array}$ \\
\hline $\begin{array}{l}\text { Physical space } \\
\text { (10 bodies) }\end{array}$ & $\begin{array}{l}8.50 \\
(1.00)\end{array}$ & $\begin{array}{r}53.67 \\
(-0.50)\end{array}$ & $\begin{array}{l}152.1 \\
(0.40)\end{array}$ & $\begin{array}{l}305.9 \\
(-0.21)\end{array}$ & $\begin{array}{l}521.2 \\
(0.28)\end{array}$ \\
\hline $\begin{array}{l}\text { Modal space } \\
\text { ( } 10 \text { bodies) }\end{array}$ & $\begin{array}{l}8.45 \\
(1.00)\end{array}$ & $\begin{array}{c}52.7 \\
(-0.51)\end{array}$ & $\begin{array}{l}152.1 \\
(0.37)\end{array}$ & $\begin{array}{l}300.5 \\
(-0.26)\end{array}$ & $\begin{array}{l}505.5 \\
(0.16)\end{array}$ \\
\hline $\begin{array}{l}\text { Modal space } \\
\text { ( } 5 \text { bodies) }\end{array}$ & $\begin{array}{l}8.52 \\
(1.01)\end{array}$ & $\begin{array}{c}54.3 \\
(-0.52)\end{array}$ & $\begin{array}{l}157.4 \\
(0.38)\end{array}$ & $\begin{array}{l}308.3 \\
(-0.20)\end{array}$ & $\begin{array}{l}512.6 \\
(0.16)\end{array}$ \\
\hline $\begin{array}{l}\text { Modal space } \\
\text { ( } 3 \text { bodies) }\end{array}$ & $\begin{array}{l}8.60 \\
(1.03)\end{array}$ & $\begin{array}{c}57.6 \\
(-0.56)\end{array}$ & $\begin{array}{l}157.6 \\
(0.37)\end{array}$ & $\begin{array}{l}337.1 \\
(-0.17)\end{array}$ & $\begin{array}{l}567.0 \\
(0.27)\end{array}$ \\
\hline
\end{tabular}

JSWE International Journal 


\subsubsection{Analysis of a group of seven bars}

Eigenvalue analysis was conducted for a fluid-coupling vibration experiment using a group of seven hexagonal bars ${ }^{(17)}$. The experimental apparatus is shown in Fig. 5. Numerical models of the structure and the fluid region for the added-mass calculation are shown in Fig. 6. Note that the added-mass matrix with respect to the nodes was conducted using a halfgroup model.

Table 2 shows the results obtained from eigenvalue analysis using the physical-space and modal-space added-mass matrices. The fifth mode is the predominant mode, where all bars vibrate in phase. The modal-space added-mass calculations gave results which matched measurements as closely as those calculated with the physical-space addedmass method. These results show that eigenvalue analysis using modal-space added mass can be used in place of that using physical-space added mass.

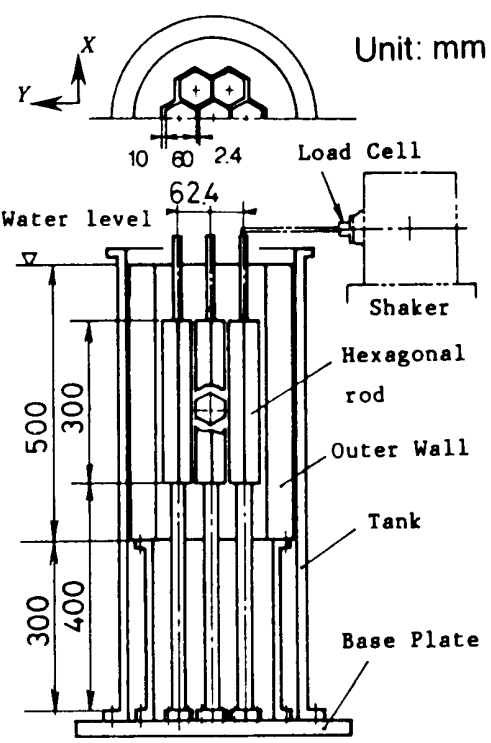

Fig. 5 Schematic of the experimental apparatus for the fluid coupling vibration experiment using a group of seven hexagonal bars

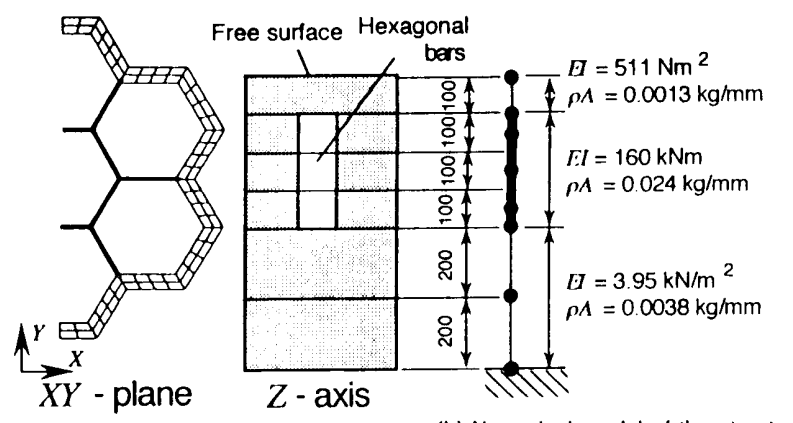

(a) Division of fluid region

(b) Numerical model of the structure

Fig. 6 Numerical model of a group of seven hexagonal bars

\section{Influence of Added-Mass Modeling on Impact-Vibration Analysis}

In this section, we compare calculation results produced using different added-mass models.

\section{1 Modeling of a bar group}

The group of 19 hexagonal bars shown in Fig. 7 is used as an example. The numerical model of the bar used here is the same as that in the model described in Sec. 2. 3. 1. The gaps between two neighboring bars are all $2.4 \mathrm{~mm}$, the modal damping ratios of each bar are $3 \%$ for the first mode and $15 \%$ for the others, the group of bars is installed in a tank of radius $180 \mathrm{~mm}$, and the nodes at the upper end have impact points which have a stiffness of $10 \mathrm{MN} / \mathrm{m}$, a damping of 0 $\mathrm{Ns} / \mathrm{m}$, and a gap of $1 \mathrm{~mm}$ (Fig. 2). We consider the fluid at the level of the tops of the bars to be in the free-surface condition in our added-mass calculations. A restraint around the group of bars limits their displacements. Calculations were made using a half-group model in consideration of the symmetry on the $Y$-direction axis. The fluid-region model

Table 2 The eigenfrequencies of fluid-coupled vibrations of a group of seven hexagonal bars

\begin{tabular}{|c||c||c|c|}
\hline Mode No. & Measured & Physical space & Modal space \\
\hline \hline 1 & - & 9.55 & 9.58 \\
\hline 2 & 9.15 & 9.64 & 9.67 \\
\hline 3 & - & 11.63 & 11.67 \\
\hline 4 & 11.85 & 12.20 & 12.25 \\
\hline 5 & 12.75 & 12.92 & 12.97 \\
\hline 6 & 13.40 & 13.53 & 13.58 \\
\hline 7 & 13.85 & 14.03 & 14.08 \\
\hline
\end{tabular}

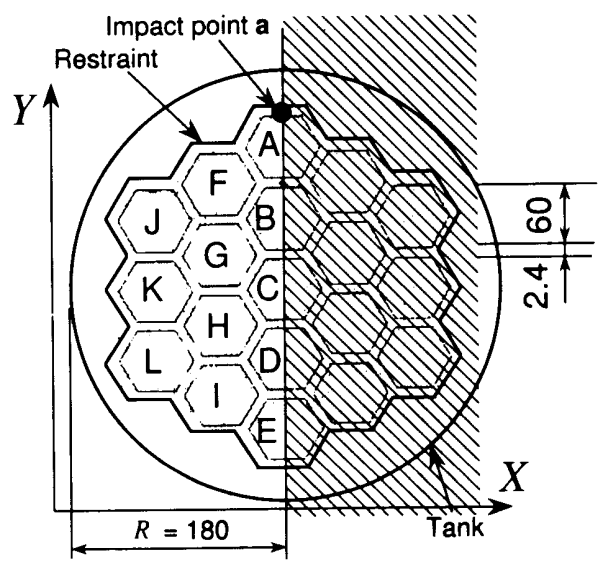

Fig. 7 A group of 19 hexagonal bars 
used for calculation of the added-mass matrix is the same as that used in the 5 -division model discussed in Sec. 2. 3. 1.

\section{2 Modeling of added mass}

Three methods for taking the fluid force of the impact vibration into consideration were compared. The first is the matrix model, which models the force using the added-mass matrix, as discussed in the previous section. The second is the concentratedmass model, which is a simplified method in which the coupling between bars is neglected and concentrated masses are introduced. This model can simulate only the predominant mode caused by base excitation. Thirdly, by neglecting the coupling between bars in the different rows, analysis using the concentrated added mass on a single row (e.g., the bars from A to $\mathrm{E}$ in Fig. 7) becomes possible when the base excitation is confined to the $Y$ direction. This will be called the single-row model. In addition, calculation without added mass was conducted for the purpose of comparison-this model will be referred to as the in air model.

The concentrated-mass evaluation was conducted using the following steps to take three-dimensional effects into account ${ }^{(17)}$. First, the equivalent addedmass coefficient $C_{v}^{*}$, based on a two-dimensional fluid region, is calculated using the method proposed by Tomita ${ }^{(8)}$. Next, the modification factor $\alpha$ is determined from the added-mass distribution and the first mode shape of the bars. Finally, the equivalent addedmass coefficient $C_{v}$ is evaluated as the product of $C_{v}^{*}$ and $\alpha$. Since the physical interpretation of $C_{v}$ is the ratio of the equivalent added-mass to the mass of the liquid displaced by the structure, the concentrated added mass can be determined. The reduction of the response of the structure due to fluid force is taken into account using the participation factor $\beta_{w}$, which can also be calculated using the coefficient $C_{v}$. For the group of bars shown in Fig. 7, the two-dimensional

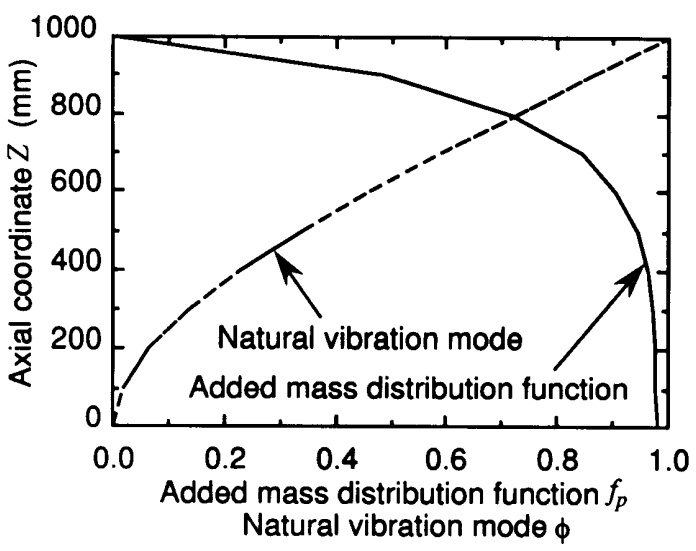

Fig. 8 Eigenmode and added mass distribution equivalent added-mass coefficient $C_{v}^{*}$ was 3.36 and the modification factor $\alpha$ was 0.72 using the eigenmode $y$ and added mass distribution $f$ shown in Fig. 8. Therefore, the values of the coefficient $C_{v}$ and the factor $\beta_{w}$ were 2.40 and 0.678 , respectively.

\section{3 Comparison of vibration characteristics}

Characteristics of vibration without impact using the above-mentioned added-mass models are compared using the acceleration-response curves at the topof-bar level in Fig. 9. In the matrix-model calculation, although the response amplitudes were slightly dependent on the positions of the bars, the resonant frequencies were almost the same. The concentratedmass model gave a similar resonant frequency and amplitude to those obtained using the matrix model. The in-air model gave a different eigenfrequency from and gave a much higher amplitude than the other models because it did not take the excitation-reduction effect into account.

\section{4 Comparison of impact vibration response}

In this section, we discuss the calculated vibration responses, including impacts, to base excitation consisting of a three-period sinusoidal wave. The excitations had an amplitude of $200 \mathrm{~cm} / \mathrm{s}^{2}$ or $400 \mathrm{~cm} / \mathrm{s}^{2}$ and a frequency of $7 \mathrm{~Hz}$ to $14 \mathrm{~Hz}$ in $1 \mathrm{~Hz}$ steps. The calculation parameters were a time step of $0.1 \mathrm{~ms}$ and a number of modes of five. In the following discussion, maximum impact forces between bar $\mathrm{A}$ and the restraint (impact point a in Fig. 7) and the maximum displacement of bar C (the central bar) are used as response values for our comparison.

3.4.1 Results using the matrix model The results using the matrix model are shown in Fig. 10, which will be used as our base of reference in the following sections.

It is observed that the response amplitude changed significantly at a certain frequency, which was larger than the linear resonant frequency of the bars

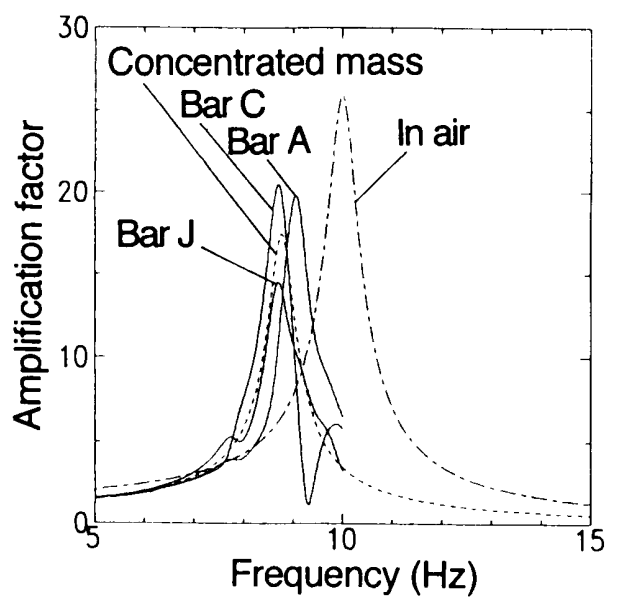

Fig. 9 Acceleration-response curves 


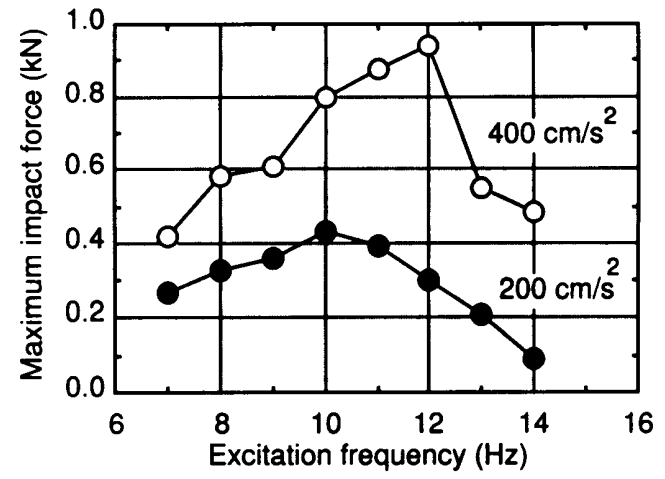

(a) Impact force at point a

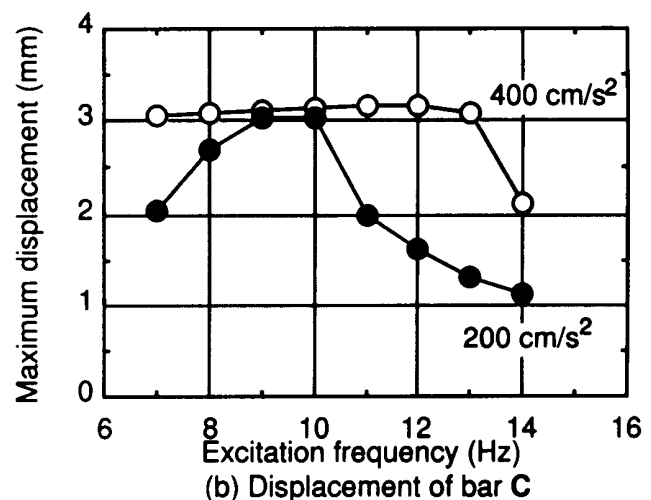

Fig. 10 Calculation results using the matrix model

$(9 \mathrm{~Hz})$. This is the jump phenomenon, a characteristic of hardening-spring nonlinear systems. The jump phenomenon was clearly observed in the $400 \mathrm{~cm} / \mathrm{s}^{2}$ excitation, in which the frequency at which the jump phenomenon occurred (jump frequency) was about 12 $\mathrm{Hz}$. In the region where impact forces were relatively large, the maximum displacement of the central bar was about $3 \mathrm{~mm}$; therefore, we consider that several bars probably contacted each other and vibrated as if they consisted of one body.

3.4.2 Comparison with the results obtained using the concentrated-mass model A comparison of results from the matrix model with these obtained using the concentrated-mass model is shown in Fig. 11. Since the jump frequency and the maximum forces around the jump frequency are in good agreement, the concentrated-mass model is considered to be effective as a simplified model. However, the impact forces at frequencies lower than the jump frequency were underestimated. This discrepancy can be understood in connection with the following reasons: Since all of the bars vibrated together as one body in resonant conditions such as $11 \mathrm{~Hz}, 400 \mathrm{~cm} / \mathrm{s}^{2}$ as shown in Fig. 12, deviation in the vibration characteristics of bars was canceled out and did not significantly influence the results. On the other hand, the bars vibrated independently at frequencies lower than the jump frequency-for example, $7 \mathrm{~Hz}, 400 \mathrm{~cm} / \mathrm{s}^{2}$

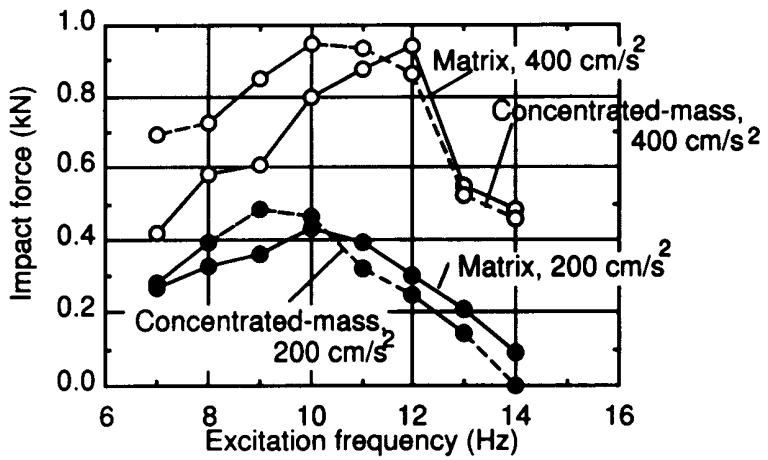

(a) Impact force at point a

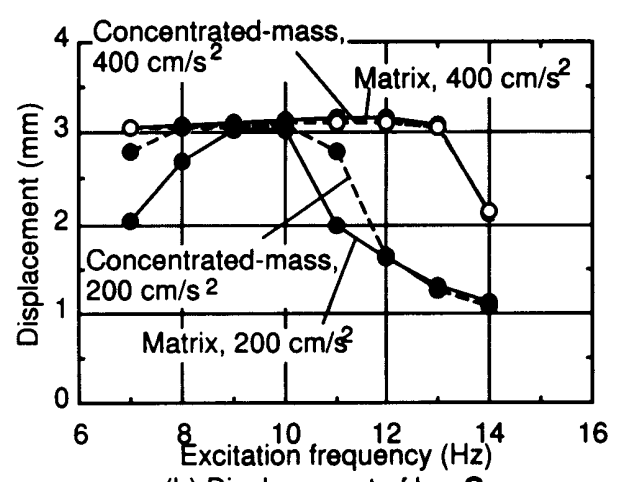

(b) Displacement of bar $\mathbf{C}$

Fig. 11 Comparison of results of the concentrated-mass model and matrix model

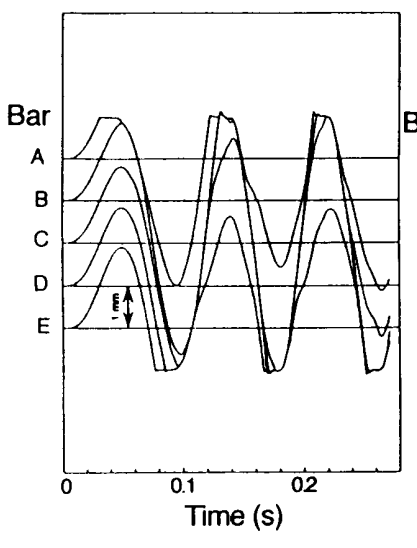

(a) Matrix model

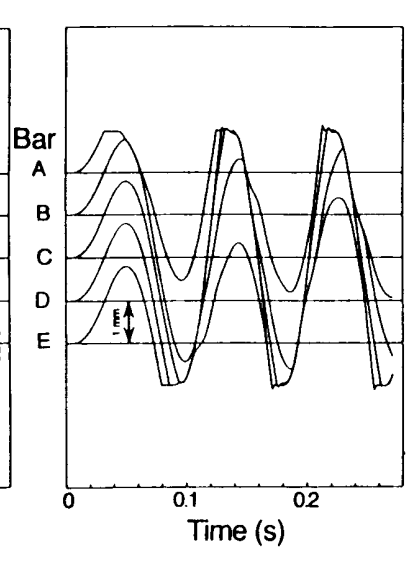

(b) Concentrated-mass model
Fig. 12 Comparison of displacement time histories (the matrix and the concentrated mass models, $11 \mathrm{~Hz}$, $400 \mathrm{~cm} / \mathrm{s}^{2}$ )

as shown in Fig. 13. Therefore, impact forces depended significantly on the vibration characteristics of the bars adjacent to the restraint.

3.4.3 Comparison with the results obtained using the in-air model A comparison of the results of the in-air model and those of our reference model is shown in Fig. 14. The responses from the former were larger than those obtained using the matrix model and the jumping frequency was higher. This difference may be due to the higher eigenfrequency of the in-air model and the fact that it does not 


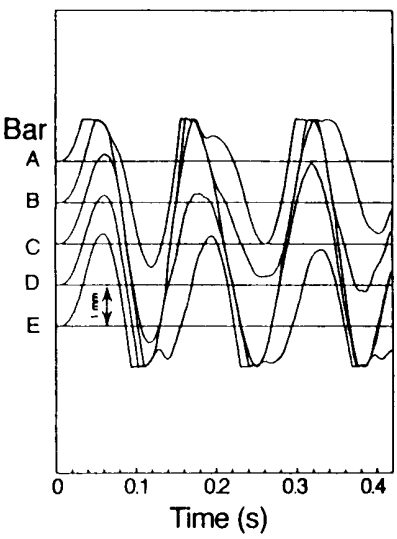

(a) Matrix model

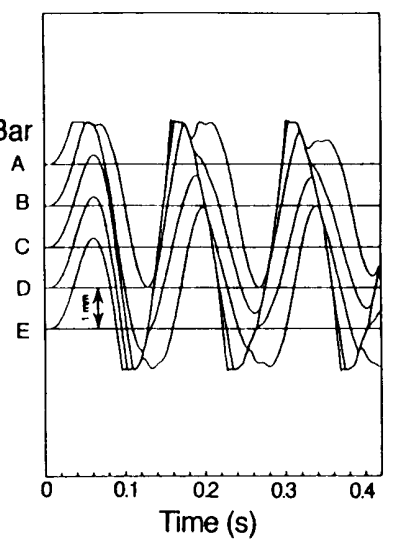

(b) Concentrated-mass model
Fig. 13 Comparison of displacement time histories (the matrix and the concentrated-mass models, $7 \mathrm{~Hz}$. $\left.400 \mathrm{~cm} / \mathrm{s}^{2}\right)$

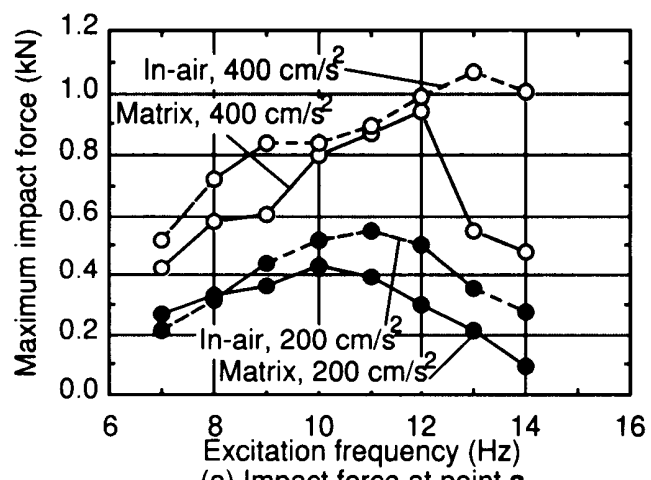

(a) Impact force at point a

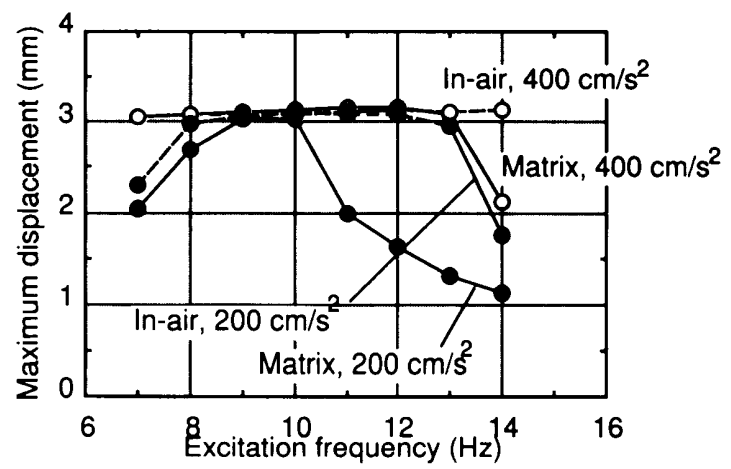

(b) Displacement of bar $\mathbf{C}$

Fig. 14 Comparison of results of the in air model and the matrix model

take the participation factor into account. Since otherwise the two models had the same parameters, the importance of evaluating the added-mass coefficient precisely when the concentrated-mass model is used was shown.

3.4.4 Comparison with the results obtained using the single-row model The results from the single-row model are shown in Fig. 15. Although the jump frequency and the maximum displacements calculated using the single-row model were close to

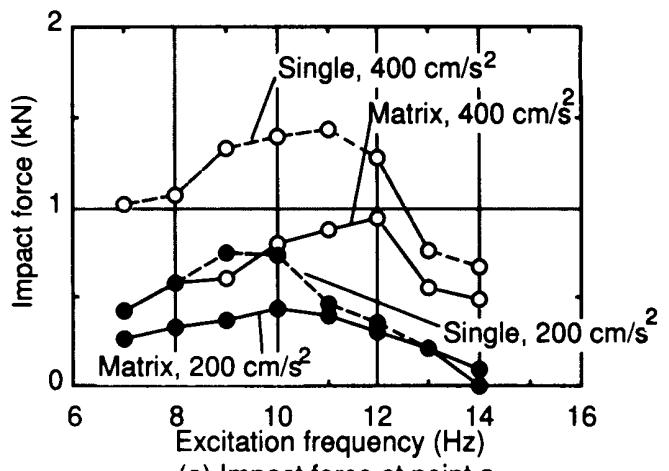

(a) Impact force at point a

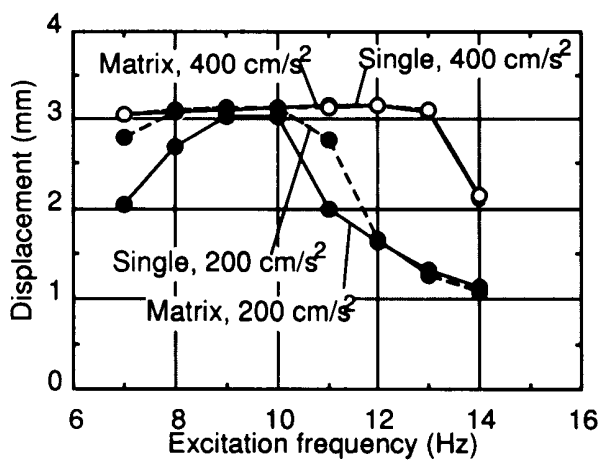

(b) Displacement of bar $\mathrm{C}$

Fig. 15 Comparison of results of the single row model and the matrix model
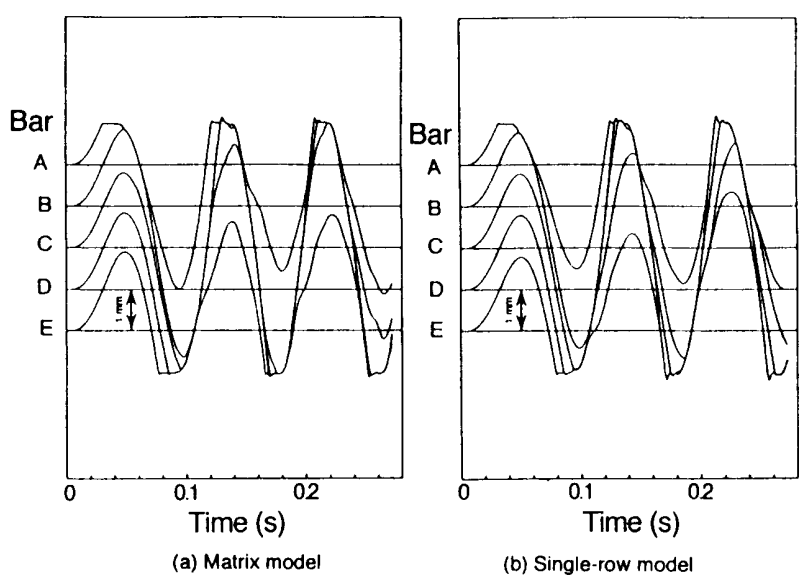

Fig. 16 Comparison of displacement time histories (the matrix and the single row models, $11 \mathrm{~Hz}, 400 \mathrm{~cm} / \mathrm{s}^{2}$ )

those obtained using the matrix model, the values of the maximum impact forces were much larger. This can be understood in the following way: The matrix model prevented the bar from vibrating as one body by taking the interaction between rows into account (e.g., the central row from bar A to bar E and the next row from bar $F$ to bar I in Fig. 7), while the single row model allowed several bars to vibrate close together and impact with the restraint as one large mass. This interpretation is supported by the time 
history shown in Fig. 16, in which the overlapped displacement lines in the single-row model appear piled up just before impacts. Therefore, as far as evaluation of vibration characteristics such as the jump frequency is concerned, the single-row model is effective.

3.4.5 Summary The displacement results and impact-force results produced using the matrix model and the concentrated mass model were close together in the resonant region; therefore, the concentratedmass method is an effective simplified model as long as the added-mass coefficient is accurately determined. However, the impact force results were not very good when the responses were small, in which cases the vibration characteristics of each bar influenced the response.

Although the impact forces calculated using the single-row model were larger than those produced using the matrix model, the single-row model is still a good simplified way to evaluate certain vibration characteristics of a group of bars, such as the jump frequency.

\section{Conclusions}

(1) As an effective method of taking fluid forces into consideration in the impact-vibration analysis of a group of hexagonal bars, a method in which an added mass matrix is introduced in the mode space of each bar was proposed. This method can reduce the number of degrees of freedom of the added-mass matrix, thus reducing the computational complexity.

(2) By applying the proposed method, the effect on the vibration response using three different methods of modeling added mass was investigated: the matrix model, in which an added-mass matrix is used for a group of bars in multiple rows ; the concentrated-mass model, in which the concentrated mass, a simplified form of added mass, is used for a group of bars in multiple rows; and the single-row model, in which concentrated mass is used for a group of bars in a single row.

\section{References}

(1) Morishita, M. and Iwata, K., Seismic Behavior of a Free-Standing Core in a Large LMFBR, Nucl. Eng. Des., Vol. 140 (1993), p. 140.

(2) Sasaki, Y., Manome, E. and Kakehi, I., Seismic Analysis of L.MFBR Core Components, Trans. 11th Int'l. Conf. Structural Mechanics in Reactor Technology, Vol. E (1991), p. 491.

(3) Preumont, A., Pay, A. and Decauwers, A., The Seismic Analysis of a Free-Standing FBR Core A, Case Study-SNR-2 Preliminary Design, Nucl.
Eng. Des., Vol. 103 (1987), p. 199.

(4) Gantenbein, F., Gauvain, J., Bernard, A. and Van Dorsselaere, J.P., Seismic Behavior of a Fast Reactor Core. Application on SUPER PHENIX 1 . Trans. 7th Int'l. Conf. Struct. Mech. in Reactor Technol., Vol. F (1983), p. 299.

(5) Stabel, J. and Kim, J.-D., Integration of Non Linear Equations of Motion, in Particular in the Context of Friction/Impact Problems, Nucl. Eng. Des., Vol. 83 (1987), p. 83.

(6) Sato, T., Imaizumi, M., Nuno, H. and Tsumura, N., The FINDS Code for Fuel Seismic Analysis Considering Inelastic Impact Behavior. ASME PVP, Vol. 150 (1988), p. 29.

(7) Shah, V.N., Bohm, G.J. and Nahavandi A.N., Modal Superposition Method for Computationally Economical Nonlinear Structural Analysis. Trans. ASME, J. Press. Vessel. Technol., Vol. 101 (1979), p. 134.

(8) Tomita, H., Flow-Path Network Method Analysis of the Vibration Characteristics of a Group of Rods in Fluid, Trans. Jpn. Soc. Mech. Eng.. (in Japanese), Vol. 49, No. 445. C (1983), p. 1474.

(9) Kasai, H., Study on the Coupled Vibration of Square Cylinders in a Liquid, Trans. Jpn. Soc. Mech. Eng., (in Japanese), Vol. 50, No.454, C (1984), p. 943.

(10) Fujita, K., Vibration Characteristics and Seismic Response of Grouped Column in Liquid. Trans. Jpn. Soc. Mech. Eng., (in Japanese), Vol. 47, No. 415, C (1981), p. 251.

(11) Shinohara, Y. and Shimogo, T., Vibrations of Square and Hexagonal Cylinders in a Liquid, Trans. ASME, J. Press. Vessel Technol., Vol. 103. No. 3 (1981), p. 223.

(12) Preumont, A., Raymackers, A. and Wyatt, M., On the Influence of the Fluid on the Seismic Response of FBR Core, Proc. of 10th Int'l Conf. on Struct. Mech. in Reactor Technol., Vol. E (1989), p. 145.

(13) Horiuchi, T., Nakagawa, M. and Kasai, H., Impact Vibration Analysis of Bar Group in Single Row, Trans. Jpn. Soc. Mech. Eng., (in Japanese), Vol. 59, No. 559, C (1993), p. 650.

(14) Horiuchi, T., Impact Vibration Analysis of Group of Hexagonal Bars, Trans. Jpn. Soc. Mech. Eng., (in Japanese), Vol. 60, No. 571, C (1994), p. 757.

(15) Zienkiewicz, O. C. and Cheung, Y. K., The Finite Element Method in Structural and Continuum Mechanics, (1967), McGraw Hill.

(16) Horiuchi, T., Nakagawa, M. and Ohashi, M., A Three Dimensional Analysis of Added Mass for Earthquake Response of Hexagonal Bars Immersed in Liquid, Trans. Jpn. Soc. Mech. Eng., (in Japanese), Vol. 59, No. 557, C (1993), p. 93.

(17) Horiuchi, T. and Nakagawa, M., Fluid Structure Interaction Vibration Characteristics of Seven Hexagonal Bars, Trans. Jpn. Soc. Mech. Eng., (in Japanese), Vol. 59, No. 557, C (1993), p. 5. 